\title{
Comparing the efficacy of intravenous ketorolac versus intravenous ibuprofen in relieving renal colic pain; a randomized clinical trial
}

\author{
Seyed Hossein Shaker ${ }^{(}$, , Seyed Ahmad Borghei $^{*}{ }^{\circledR}$ \\ Emergency Medicine Department, Iran University of Medical Science, Tehran, Iran
}

\section{A R T I C L E I N F O}

Article Type:

Original

\section{Article History:}

Received: 7 June 2018

Accepted: 11 July 2018

ePublished: 29 July 2018

\section{Keywords:}

Ketorolac

Ibuprofen

Renal colic

Renal stone

\begin{abstract}
A B S T R A C T
Introduction: Various therapies have been used to relieve or improve the urinary colic pain, but many of these treatments have been limited due to side effects associated with the drugs. In this regard, opioid drugs and nonsteroidal anti-inflammatory drugs (NSAIDs) have a higher priority than other treatments.

Objectives: The aim of the present study was to compare the level of pain relief from renal stones after the administration of two types of NSAIDs, including intravenous ibuprofen versus intravenous ketorolac.

Patients and Methods: In this clinical trial (identifier: IRCT20180108038276N1), 70 patients with renal colic pain were randomly treated with intravenous ketorolac $(30 \mathrm{mg}, \mathrm{n}=35)$ or with intravenous ibuprofen ( $800 \mathrm{mg}, \mathrm{n}=35$ ). The patient's pain severity was assessed by the visual analogue scaling (VAS) method before and 20 and also 60 minutes after interventions.

Results: Although pain severity considerably reduced within 60 minutes of prescribing ibuprofen and ketorolac, the trend of the change in pin score was not different between the two groups $(P=0.734)$. We found no significant difference in appearance of some side effects including nausea and vomiting, requiring use of ondansetron, need to prescribe additional analgesics. However epigastric pain and need to administrating $\mathrm{H} 2$ blockers were not revealed in the group receiving ketorolac, but these complications were found in $25.7 \%$ of the patients received ibuprofen indicating a significant difference $(P=0.002)$.

Conclusion: A single dose administration of both intravenous ketorolac and ibuprofen is associated with significant improvement in renal colic pain within one hour after administration. However, due to the lower incidence of gastrointestinal complications in the administration of ketorolac compared to ibuprofen, the use of ketorolac in treatment and relief of renal colic pains is preferable.
\end{abstract}

Implication for health policy/practice/research/medical education:

In this clinical trial, on 70 patients with renal colic pain which were randomly treated with intravenous ketorolac $(30 \mathrm{mg}, \mathrm{n}=$ 35 ) or with intravenous ibuprofen $(800 \mathrm{mg}, \mathrm{n}=35)$, we found that single dose administration of both intravenous ketorolac and ibuprofen is associated with significant improvement in renal colic pain within one hour after administration. However, due to the lower incidence of gastrointestinal complications in the administration of ketorolac compared to ibuprofen, the use of ketorolac in treatment and relief of renal colic pains is preferable.

Please cite this paper as: Shaker SH, Borghei SA. Comparing the efficacy of intravenous ketorolac versus intravenous ibuprofen in relieving renal colic pain; a randomized clinical trial. J Nephropharmacol. 2018;7(2):122-125.

\section{Introduction}

Renal stone disease or urolithiasis is a very common disease in different centuries (1). The renal colic is characterized by forming an acute pain with a colic nature in the flanks due to stones formation in the renal tubules, especially the urethra. Classical renal colic manifestations include the pain of the flank to the groin area with hematuria (in about $85 \%$ of cases) with nausea and vomiting. Renal colic pain is often described as the most severe pain experienced by an individual (2-4). As a result, the use of analgesics such as nonsteroidal anti-inflammatory drugs (NSAIDs) and opioids, or by combining with other drugs, such as antispasmodics plays an important role in the treatment of renal colic pains. Recently, the use of alphablockers (with decreasing transit time for urinary tract stones), nifedipine, intravenous lidocaine, and neuronal 
block have also led to a decrease in renal pain $(5,6)$. Narcotics have been used to control colic pain for many years $(7,8)$. Although narcotics such as morphine, codeine and meperidine have been very useful for improving renal colic pain, these drugs have little effect on the underlying cause of these pains, such as prostaglandins. The benefits of using opioids include low-cost, high effectiveness, and the desirable likelihood of titration. However, most physicians do not tend to use these drugs because of side effects such as nausea and vomiting, sedation, shivering, light stomach, narcotic dependence, disturbances, respiratory depression, and hypotension (8).

The NSAIDs have been used alone or in combination with opioids for the treatment of renal pains for a long time (7). The analgesic effects of these drugs were due to inhibiting the synthesis of prostaglandins. As a general result, NSAIDs inhibit vasodilatation of the renal arteries and increase vascular permeability, resulting in diuresis and increased pressure on the urethra. NSAIDs also reduce the edema, inflammation and hyperactivity of the ureter muscle (9). The effects of NSAIDs on pain relief in acute renal colic have been similar to opioids. The only limitation of NSAIDs compared to opioids is the delay in initiating their analgesic effects with intravenous opioids.

\section{Objectives}

The aim of this study was to evaluate the effects of the two types of NSAIDs including intravenous ibuprofen and intravenous ketorolac in patients with renal colic.

\section{Patients and Methods}

\section{Study population}

In this study, all patients who were referred to one of the educational hospitals of Iran University of Medical Sciences due to renal colic with any age and gender were included. Patients with cardiovascular disease, blood pressure crisis, or diabetes were excluded from the study. Written consent was obtained from patients prior to entering the study. The purpose of the research project was explained for the participants. In this study, the patient's pain severity was first measured by visual analogue scaling (VAS) score. Then, the patients were randomly assigned to two groups (using the random number table) receiving intravenous ibuprofen $(800 \mathrm{mg})$ or intravenous ketorolac $(30 \mathrm{mg})$. After 20 and 60 minutes, patients were again asked for pain severity with the same criteria before receiving the drug. All information such as demographic and clinical information such as renal colic history as well as drug history were collected through interviews from patients and recorded in the checklist. The study endpoint was to assess and compare the pain severity before and after study therapeutic protocols in each patient's subgroups.

\section{Ethical issues}

Human rights were respected in accordance with the
Helsinki Declaration 1975, as revised in 1983. The ethical committee of Iran University of Medical Sciences (Ethical No. IR.IUMS.FMD.REC.1396.9411307007) approved the study. The informed consent was taken from the patients as well as from parents and first relatives. The study was also registered in the Iranian Registry of Clinical Trials (identifier: IRCT20180108038276N1, https://www.irct. ir/trial/28954). Additionally, this paper was extracted from the residential thesis of Seyed Ahmad Borghei with number 2812 in Iran University of Medical Sciences.

\section{Statistical analysis}

For statistical analysis, results were presented as mean \pm standard deviation (SD) for quantitative variables and were summarized by absolute frequencies and percentages for categorical variables. Normality of the data was analyzed using the Kolmogorov-Smirnoff test. Categorical variables were compared using chi-square test or Fisher's exact test when more than $20 \%$ of the cells with expected count of less than 5 were observed. The quantitative variables were also compared with $t$ test or Mann-Whitney $\mathrm{U}$ test. For the statistical analysis, the statistical software SPSS version 16.0 for Windows (SPSS Inc., Chicago, IL) was used. $P$ values of 0.05 or less were considered statistically significant.

\section{Results}

In this study, 35 patients with renal colic pain were treated with intravenous ketorolac (30 $\mathrm{mg}$ ) and 35 patients treated with intravenous ibuprofen $(800 \mathrm{mg})$. The two groups were matched in terms of average age, gender distribution, and history of renal colic pain (Table 1). As shown in Table 1, there was no difference in pain severity between the groups before intervention and 20 and also 60 minutes after medication. According to the repeated measure ANOVA test, although pain severity considerably reduced within 60 minutes of prescribing ibuprofen and ketorolac, the trend of the change in pain score was not different between the groups $(P=0.734)$ (Figure 1). Regarding drug-related side effects (Figure 2), we found no significant difference of various side effects including nausea and vomiting, requiring use of ondansetron and need to prescribe additional analgesics. While epigastric pain and need to administrating of $\mathrm{H}_{2}$ blockers were not revealed in the group receiving ketorolac, these

Table 1. Baseline characteristics of study population

\begin{tabular}{lccc}
\hline Item & $\begin{array}{c}\text { Ketorolac group } \\
(\mathbf{n}=\mathbf{3 5})\end{array}$ & $\begin{array}{c}\text { Ibuprofen group } \\
(\mathbf{n}=\mathbf{3 5})\end{array}$ & $\boldsymbol{P}$ value \\
\hline Mean age, year & $36.51 \pm 11.64$ & $38.29 \pm 11.71$ & 0.528 \\
Gender & & & 0.324 \\
Male & $31(88.6 \%)$ & $28(80.0 \%)$ & \\
Female & $4(11.4 \%)$ & $7(20.0 \%)$ & \\
History of renal stone & $18(51.4 \%)$ & $22(62.9 \%)$ & 0.334 \\
\hline
\end{tabular}




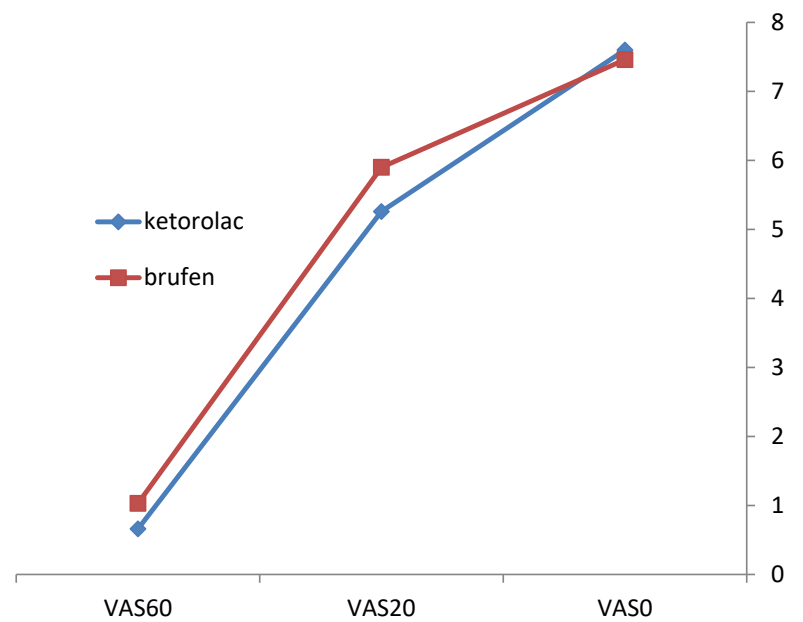

Figure 1. The trend of the change in pain score in the two treatment groups.

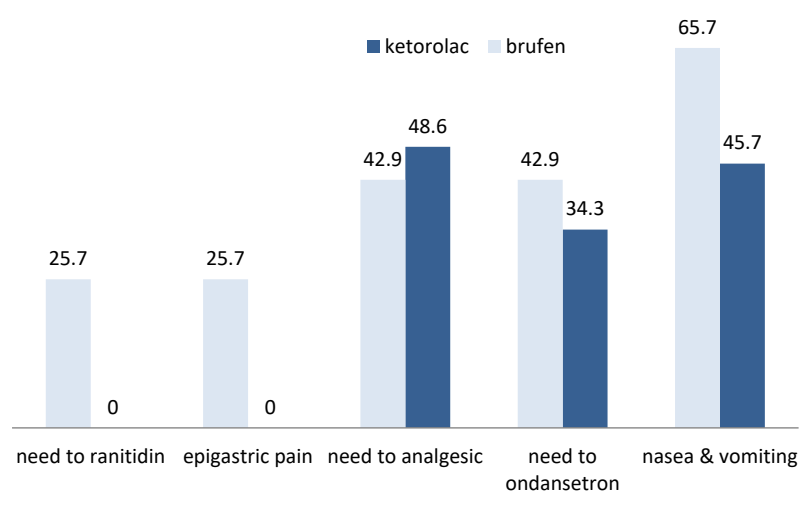

Figure 2. The frequency of drug-related side effects in the two treatment groups.

complications were found in $25.7 \%$ of the patients received ibuprofen indicating a significant difference $(P=0.002)$.

\section{Discussion}

Various therapies have been used to relieve or improve the urinary colic pain, but many of these treatments have been limited due to side effects associated with the drug. In this regard, opioid drugs and NSAIDs have a higher priority than other treatments. However, serious side effects such as hypotension, respiratory depression and gastrointestinal bleeding, as well as increased risk of coronary artery disease, or an increased risk of renal or cardiac failure, have prevented the selection of the best treatment regimen. In this regard, the use of NSAIDs with various forms and doses has been studied in recent years. What we assessed in this study was a comparison of the improvement in pain relief from kidney stones after the administration of two types of these drugs, including intravenous ibuprofen and ketorolac. In the current clinical trial, these drugs were injected in a single dose of 30 and $800 \mathrm{mg}$, respectively, and the severity of the colic pain at 0,20 and 60 minutes after injection was evaluated and compared, as well as the adverse effects associated with the administration of drugs were also determined. First, our study found, no significant difference on the effect of the two drugs to reduce renal colic pain. In other words, in both regimens, although the reduction in the severity of pain was evident within 60 minutes of treatment, the superiority of a regimen to the other regimen was not detected. Therefore, regarding the efficacy and therapeutic effectiveness, no difference between the two drugs was seen. Although no difference between the groups in terms of the frequency of nausea and vomiting and the need for administration of ondansetron and also the need for additional doses of opioids was detected, however, the prevalence of epigastric pain and the consequent need for administration of ranitidine in the group treated with ibuprofen was far higher than that of the ketorolac group. In fact, in the ketorolac group, no complication of pain or epigastric discomfort was reported. Therefore, regarding safety, ketorolac is superior to ibuprofen, and in general, the administration of ketorolac will be preferable to improve real colic pain.

Although most of the studies have emphasized the high efficacy and safety of ketorolac versus other NSAIDs. In the study by Larsen et al (10), in patients treated with intravenous ketorolac ( $30 \mathrm{mg}$ ), a median pain score of 9 at intervention time reduced to score 1 about 30 minutes after intervention with no drug-related adverse effects which was perfectly in accordance with our study. In Neighbor and Puntillo's trial (11), there was no difference between the two groups receiving intravenous ketorolac and intravenous ibuprofen in terms of severity of pain during the study period. Likewise, both groups had a significant reduction in pain, which was completely consistent with our study. However, it is imperative that by choosing the best dose of the drugs to be studied, in particular for ketorolac, in addition to increasing the chances of relieving colic pain, the side effects of drugs including nausea and vomiting and the need for supplemental analgesics can be also minimized.

Although ketorolac is effective in reducing colic pain, some side effects should always be taken into account. In various studies, there was an increased risk of exacerbation of ischemic heart disease and the risk of myocardial infarction. In general, the administration of this drug in the following cases should be very cautious; having allergy to ketorolac, peptic ulcer disease, bleeding disorders or coagulation disorders, history of trauma to the brain or cerebrovascular accidents, bleeding during recent surgery, severe renal disease or history of dehydration, history of asthma or severe allergic reaction after taking aspirin or NSAIDS, pregnancy or lactation, as well as history of any ischemic heart disease.

\section{Conclusion}

In the final conclusion, it can be concluded that single 
dose administration of both intravenous ketorolac and ibuprofen is associated with significant improvement in renal colic pain within one hour after administration. However, due to the lower incidence of gastrointestinal complications in the administration of ketorolac compared to ibuprofen, the use of ketorolac in treatment and relief of renal colic pains is preferable.

\section{Study limitations}

During the research, we encountered some problems such as inconsistencies in implementation and time constraints.

\section{Authors' contribution}

SHH and BA designed the study, observed accuracy and validity of the study. BA collected the data and follow the study. $\mathrm{SHH}$ and BA supervised the project. $\mathrm{SHH}$ and BA wrote the paper. All authors edited and revised the final manuscript and accepted its publication.

\section{Conflicts of interest}

The authors declared no competing interests.

\section{Ethical considerations}

Ethical issues (including plagiarism, data fabrication, double publication) have been completely observed by the authors.

\section{Funding/Support}

The authors declare that there is no source of funding for the research reported.

\section{References}

1. Portis AJ, Sundaram CP. Diagnosis and initial management of kidney stones. Am Fam Physician. 2001; 63:1329-38.

2. Iguchi M, Katoh Y, Koike H, Hayashi T, Nakamura M. Randomized trial of trigger point injection for renal colic. Int J Urol. 2002; 9:475-9.

3. Bektas F, Eken C, Karadeniz O, Goksu E, Cubuk M, Cete Y. Intravenous paracetamol or morphine for the treatment of renal colic: a randomized, placebo- controlled trial. Ann Emerg Med. 2009;54:568-74. doi: 10.1016/j.annemergmed.2009.06.501.

4. Edwards JE, Meseguer F, Faura C, Moore RA, McQuay HJ. Single dose dipyrone for acute renal colic pain. Cochrane Database Syst Rev. 2002;(4):CD003867. DOI: 10.1002/14651858.CD003867

5. Soleimanpour H, Hassanzadeh K, Vaezi H, Golzari SE, Esfanjani RM, Soleimanpour M. Effectiveness of intravenous lidocaine versus intravenous morphine for patients with renal colic in the emergency department. BMC Urol. 2012;12:13. doi: 10.1186/1471-2490-12-13

6. Dellabella M, Milanese G, Muzzonigro G. Randomized trial of the efficacy of tamsulosin, nifedipine and phloroglucinol in medical expulsive therapy for distal ureteral calculi. J Urol. 2005; 174:167-72. doi: 10.1097/01.ju.0000161600.54732.86

7. Phillips E, Hinck B, Pedro R, Makhlouf A, Kriedberg $\mathrm{C}$, Hendlin K, et al. Celecoxib in the management of acute renal colic: a randomized controlled clinical trial. Urology. 2009;74:994-9. Doi: 10.1016/j. urology.2009.04.063

8. Safdar B, Degutis LC, Landry K, Vedere SR, Moscovitz HC, D’Onofrio G. Intravenous morphine plus ketorolac is superior to either drug alone for treatment of acute renal colic. Ann Emerg Med. 2006; 48:173-81.

9. Serinken M, Karcioglu O, Turkcuer I, Ozkan HI, Keysan MK, Bukiran A. Analysis of clinical and demographic characteristics of patients presenting with renal colic in the emergency department. BMC Res Notes. 2008; 1:79. Doi: 10.1186/1756-0500-1-79

10. Larsen LS ${ }^{1}$, Miller A, Allegra JR. The use of intravenous ketorolac for the treatment of renal colic in the emergency department. Am J Emerg Med. 1993;11:197-9.

11. Neighbor ML, Puntillo KA. Intramuscular ketorolac vs oral ibuprofen in emergency departmentpatients with acute pain. Acad Emerg Med. 1998;5:118-22.

Copyright $\odot 2018$ The Author(s); Published by Society of Diabetic Nephropathy Prevention. This is an open-access article distributed under the terms of the Creative Commons Attribution License (http://creativecommons.org/licenses/by/4.0), which permits unrestricted use, distribution, and reproduction in any medium, provided the original work is properly cited. 